\title{
No Pacing
}

National Cancer Institute

\section{Source}

National Cancer Institute. No Pacing. NCI Thesaurus. Code C95877.

Problem associated with the device ceasing to deliver paces. 